\title{
Labor Pain Mangement Practice and Associated Factors Among Obstetric Care Workers in Public Health Facilities of West Guji Zone, Southern Ethiopia, 2020
}

Girma Tufa Melesse ( $\square$ tgirma281@gmail.com )

BHU: Bule Hora University

Zelalem Jabessa Wayessa

BHU: Bule Hora University

Amana Jilo Bonkiye

BHU: Bule Hora University

\section{Research}

Keywords: Labor, pain management practice, West Guji Zone, Ethiopia

Posted Date: October 6th, 2021

DOl: https://doi.org/10.21203/rs.3.rs-936593/v1

License: (c) (i) This work is licensed under a Creative Commons Attribution 4.0 International License.

Read Full License 


\section{Abstract}

Introduction: Childbirth is a painful experience for almost all women and most women require pain relief. Labor pain management strategies include non-pharmacological interventions and pharmacological interventions. Globally, approximately 140 million births occur every year and most of them are vaginal births among pregnant women with no risk factors identified for complications during onset of labor.More than a third of maternal deaths from pregnancy-related conditions are attributed to complications that arise during childbirth or the period immediately following childbirth, usually due to bleeding, obstructed laboror sepsis. The aim of this study was to assess labor pain management practice and associated factors among obstetric care givers in public health facilities of West Guji, Southern Ethiopia 2020.

Methods: Institutional based cross-sectional study was conducted. Simple random sampling technique was employed to select 280 study participants. The data was collected by using self-administered questionnaire. Descriptive statistics, binary and multiple logistic regressions were used. Adjusted odds ratios (AOR) with $95 \% \mathrm{Cl}$ was estimated to assess the strength of associations and statistical significance was declared at a p-value $<0.05$.

Result: The magnitude of labor pain management practice among obstetric care workers at the study area was $43.3 \%$. Factors that associated to labor pain management practice which were found to be statistically significant by multivariable analysis model were: Being diploma holder (AOR, 0.3; 95\% Cl: 0.2, 0.6), knowledge of obstetric analgesia (AOR, 9.4; $95 \% \mathrm{Cl}: 1.8,9.9)$ andthought of pharmacologic obstetric analgesia has effect on the labor (AOR, 2.9; $95 \% \mathrm{Cl}: 1.4,6.2)$

Conclusion: Though the magnitude of labor pain management practice was better than other studies, stillit needs working to increase it. Being diploma holder, knowledge of obstetric analgesia and thought of pharmacologic obstetric analgesia has effect on the labor were the factors that responsible for labor pain management practice.

\section{Background}

Labor is the term for the changes in anatomy and physiology in the female reproductive tract that prepare the fetus and the placenta for delivery. In the majority of cases, this happens when the baby is fully developed at full term, between 37-40 weeks of gestation. Labor heralds the end of the baby's time in the uterus and the beginning of adaptation to life outside the mother(1).

Childbirth is a painful experience for almost all women. The pain that women experience during labor is affected by multiple physiological and psychosocial factors and its intensity can vary greatly. Most women in labor require pain relief. Labor pain management strategies include non-pharmacological interventions (that aim to help women cope with pain in labor) and pharmacological interventions (that aim to relieve the pain of labor)(2). 
The management of pain during labor involves more than the act of administering the best anesthetic agent available in a timely fashion. The type of pain relief should always be individualized after a complete discussion with each woman. Every woman should enter the labor process fully aware of all available pain relief options in the health care setting in which they will give birth. It is the responsibility of every health care provider (and that health care providers inform women) about all approaches, including advocating for access to pain relief in the health care facility(3).

Childbirth experience has been influenced by many factors such as knowledge about the labor process, quality of care and services, severity of labor pain, method of labor pain management and medical interventions. Medical interventions that result from the advancement of medical science and seek to ensure the safety of childbirth sometimes eliminate the concept of labor as a physiologic phenomenon and look to it as a medical problem(4).

Globally, approximately 140 million births occur every year and most of them are vaginal births among pregnant women with no risk factors identified for complications during onset of labor(5).More than a third of maternal deaths from pregnancy-related conditions are attributed to complications that arise during childbirth or the period immediately following childbirth, usually due to bleeding, obstructed laboror sepsis (6).

Pain can have negative effects on the mother and fetus. The psychological effects of severe pain should not be overlooked, especially in cases where they are associated with an adverse fetal or maternal outcome(7). Different pain control measures have varying degrees of success and side effects for women and children. In some European countries, doctors generally prescribe inhaled nitrous oxide gas for pain control, particularly $53 \%$ nitrous oxide and $47 \%$ oxygen in the UK; midwives can use this gas without a prescription (8).

Developing countries requires high levels of training; staff and technology that make its applicability of labor analgesia effective and staff will have little to no training in advanced drug techniques, such as epidurals and the use of intravenous or neuraxial opioid drugs. They will not have confidence in starting these techniques and will not be aware of possible complications. There is often a widespread fear that the use of opioids for acute pain quickly leads to addiction(9). Pain during childbirth is complex and is considered a unique and individual experience. Pain management requires a flexible approach with several options so that a woman can choose what works for her (10).

The World Health Organization (WHO) includes pain management as a quality standard of care, emphasizing that all aspects of health care must be provided in a timely, adequately manner and must respect choice, culture and need a woman (11). The degree of pain and the ability of each woman to deal with it depend on several factors: when women are properly prepared and supported, they produce endogenous analgesic substances that help them work with the pain of labor (12). Most healthcare professionals are aware of several approaches to pain management, including pharmacological and nonpharmacological options. Challenges included shortages of staff, lack of equipment, access to nitrous oxide or epidural drugs and fears about the effect of opioids on women and / or children(13). 
According to a study conducted in Tanzania, it shows that the barriers affecting the provision of pain relief options are barriers to the health system (lack of staff, equipment and protocols), limited education and opportunities to practice pain relief methods and beliefs negatively, fears and bad practices(14). The pain experienced during labor has multiple physiological and psychosocial dimensions and its intensity can vary greatly from one woman to another. The most common drug treatment for pain included in the labor ward is pethidine (15) and nonpharmacological management methods include massages, walks, showers and music, as well as various positioning techniques to help the mother manage the discomfort. Many women are able to control their pain with nothing but education and these non-invasive methods of pain control(16).

The opioid is more effective in early active labor and less effective after seven centimeters of dilation and morphine is preferred over pethidine due to its reduced half-life in women and children, and pethidine may be associated with reduced success in breastfeeding due to the child's reduced ability to initiate and support breastfeeding(17).

In Ethiopia, the study showed that the prevalence of analgesia used during childbirth was $37.9 \%$. Reliving the pain of labor is a major concern of pregnant women, family members and healthcare professionals (18). Another study conducted in the Amhara region indicates that $34.4 \%$ of healthcare professionals used pain relief during childbirth (19). Studies have shown that several obstacles to the use of labor analgesia include obstacles related to healthcare professionals, obstacles to the system and obstacles to the patient. Effective pain relief during labor is not only attentive, but has enormous physiological and psychological benefits for both mother and baby (20).

However, pharmacological and non-pharmacological interventions are reference standards to relieve pain during labor and delivery; yet, utility and practice were scarce, especially in developing regions such as Ethiopia. Therefore, this study was aimed to assess the magnitude of labor pain management practice and the associated factors among obstetric care givers in public health facilities of west Guji zone. This study will help us to assess the magnitude of labor pain management practice and associated factors among health care workers in public health facilities of West Guji zone. Thus the labor pain is the worst pain; the management of labor pain during normal labor improves feto-maternal outcome and satisfaction of laboring women.

It will support policy makers, health planners and managers in drafting guidelines and provide guidance on managing pain at labor. It also helps obstetric care professionals to provide prenatal education on the need for methods to relieve pain during labor, both pharmacological and non-pharmacological methods during childbirth. It will identify the challenges of labor pain management, method to relieve pain at labor and factors influencing pain management at labor to improve institutional delivery to achieve the goal of sustainable development, reduce maternal mortality and morbidity and prevent the death of newborns through the use of services provided by a qualified obstetric care givers. In addition, this study will serve as input for the future researcher. 


\section{Methods}

\section{Study area and Study period}

The study was conducted in West Guji Zone, Oromia Region, Southern Ethiopia. West Guji zone is located $467 \mathrm{Km}$ far from Addis Ababa to the south direction at $5^{\circ} 35^{\prime} \mathrm{N}$ Latitude and $38^{\circ} 15^{\prime} \mathrm{E}$ Longitude. The zone has 9 woredas and 196 kebeles, of these 166 are the rural and 30 are the urban kebeles. It has an estimated population of 1,389,821 of whom 681,012 are male and 708,809 are females. West Guji Zone has 1 general Hospital, 2 primary hospitals, 42 health centers and 166 health posts, and has 860 health care providers and 478 health extension workers. The study was conducted from July 11/2020 to May 30, 2021.

\section{Study design}

Institutional based cross-sectional study design was conducted to assess labor pain management practice and associated factors among Obstetric care workers of West Guji Zone.

\section{Source population}

The source population were all health care workers found in West Guji Zone public health facilities

\section{Study population}

The study population were all obstetric care givers available during the study period in West Guji Zone public health facilities

\section{Inclusion criteria}

All obstetric care givers (Doctors, Midwives, Nurses, Health Officers and Anesthetists) who were giving obstetric care in the delivery room

\section{Exclusion criteria}

All obstetric care workers who were newly recruited within the last six months

\section{Sample Size Determination}

Sample size was calculated for each objective and optimal sample size was selected. For objective one, sample size was determined using single population proportion formula with the following assumptions: the proportion of labor pain management practice which is taken from the previous study done in Tigray General Hospitals, Northern Ethiopia.

Formula, $n=(Z a / 2)^{2} p(1-p) / d 2$, where $n=(1.96)^{2}(0.433)(0.566) /(0.05)^{2}=377$

Where; $\mathrm{n}=$ sample size 
$d=$ margin of error $(5 \%)$

$p=$ proportion of labor pain management practice $(43.3 \%$ or $p=0.433(37)$.

$\mathrm{Z} / 2$ = critical value at $95 \% \mathrm{Cl}$ is 1.96.

By adding $10 \%$ of non-response rate $377+10 \%=415$

Sample size was also calculated for objective two using double population proportion formula by Epi-Info version 7 , by using type I error $5 \%$,power of $80 \%$ and ratio of exposed to un exposed $1: 1$ and $95 \% \mathrm{Cl}$ from previous study conducted in Tigray General Hospitals(37).

Table 1

Sample size determination for factors associated with labor pain management practice of West Guji Zone public health facilities, Oromia region, Ethiopia, 2020.

\begin{tabular}{|llllllll|}
\hline S/No & Variable & Cl & Power & $\begin{array}{l}\text { Prevalence } \\
\text { among } \\
\text { unexposed }\end{array}$ & AOR & $\begin{array}{l}\text { Non } \\
\text { response } \\
\text { rate }\end{array}$ & $\begin{array}{l}\text { Total } \\
\text { sample } \\
\text { size }\end{array}$ \\
\hline 1 & $\begin{array}{l}\text { Professionals positive } \\
\text { attitude for labor pain } \\
\text { management }\end{array}$ & $95 \%$ & 80 & $56.7 \%$ & 2.242 & $10 \%$ & 266 \\
\hline 2 & High level qualification & $95 \%$ & 80 & $50.0 \%$ & 2.876 & $10 \%$ & 154 \\
\hline
\end{tabular}

Based on the above formula sample size computed for both objectives and the largest sample size has been taken which is obtained from objective 1 (415). Since the total population of the study area is less than 10,000 , correction formula was used.

Hence, $n / 1+(n / N)=415 / 1+(415 / 860)=280$ subjects

\section{Sampling procedure}

Among the total 3 Public hospitals and 42 health centers in the Zone, all hospitals were selected purposefully and 15 health centers were selected by simple random sampling technique. Hospitals selected for study were Bule Hora General Hospital, Karcha Primary Hospital and Malka Soda Primary Hospitals with 154, 50 and 33 obstetric care givers respectively.

Health centers selected for this study were Q/Rasa, Garba, Ela Farda, G/Soke, Ela Dima, Dimtu, Bore, Guwanguwa, Bukisa, Finchawa,G/Dibisa,Bule Hora,Shara, Suro and Soda with a total of 294 obstetric care givers. The required number of study subjects were proportionally allocated to each health facility according to the number of health care providers in each health facility. From all Hospitals 185 obstetric care givers were selected and from all health centers 230 obstetric care givers were selected by proportional allocation to sample size. Finally, Simple random sampling technique was employed to select 415 study participants. 


\section{Dependent variable}

\section{Labor pain management practice \\ Independent variables}

\section{Socio-demographic factors}

Age, Sex, Residence, Ethnicity, Religion, Profession, qualification of HCPs, Clinical experience in year

\section{Obstetric care giver factors}

knowledge, attitude, pain expectation, caregiver preferences.

Institutional factors: - availability of analgesic agents, equipment, privacy, number of staff.

\section{Operational definition}

\section{Labor}

a continuous process in which progressive regular uterine contractions result in the expulsion of the fetus.

\section{Obstetric care givers}

skilled health professionals who are giving maternal care service in the delivery room.

\section{Data collection tools}

The data was collected by using a pretested structured self-administered questionnaire with data collector guidance. The tool is adapted from different studies considering the local situation of the study area and purpose of the study and it was prepared in English language to be understood by every respondent.

\section{Data collection Procedure}

Eighteen BSc Nurses (one from each health facility) were recruited as data collectors, nine assistant lecturers will be recruited as supervisors from Bule Hora University College of health and Medical sciences and trained for two days about data collection. Self-administered structured questionnaire was delivered to each obstetric care giver professionals in the delivery room during the data collection period and requested to fill the data honestly.

\section{Data quality control}

To control the quality of data, structured questionnaire was used, and pre-test was done on $5 \%$ of the study population at Adola General Hospital to ensure the clarity and applicability of the tool and then necessary modification has been done on age. 
Data collectors along with the supervisors were trained for two days regarding to the purpose of the study, study tools, data collection procedures, how to interview and check the questionnaires for completeness during collecting data, and how to handle the collected data.

Supervisor was carried out his/her regular supervision, spot-checking and reviewing the completed questionnaire daily to maintain data quality and necessary feedback offered to the data collectors. The overall activity was coordinated by the principal investigator.

\section{Data processing and analysis}

Data was entered into Epi data 3.1 version and data cleaning (checking for missing values, recoding and outliers) was done after exported to SPSS version 25 statistical software analysis. For further analysis cross tabulation was done to identify associations among dependent and independent variables for data exploration and to compute descriptive summary statistics.

The descriptive statistics result of numerical variables expressed by mean with standard deviation while categorical variables was presented using frequency with percentage, and displayed by tables, bar graph, line graph and pie chart, whereas the normality of continuous variables was checked by histogram.

Bivariable logistic regression analysis was used to assess the association between the dependent and all the independent variables and to identify candidate variables for multivariable analysis at $p$-value $<0.25$ in order to control potential confounders and to predict the association of explanatory variables and outcome.

Multivariable logistic regression analysis was applied by considering the selected variables of bivariable logistic regression analysis to identify statistically significant at $P<0.05$. The Hosmer and Lemeshow model goodness of fit test was checked for the fitness of the variables fulfilled at $p$-value $>0.05$. Multicollinearity was tested among independent variables that have similar effect on the outcome variable.

\section{Ethical considerations}

The study protocol was approved by Research and Publication Directorate, Bule Hora University. Based on the approval, an official letter was written by Research and Publication Directorate to West Guji Zone Administration Office and Health Department. Explanation on the objective of the research was provided to the concerned personnel at each level. The Zonal Health Department wrote the letter to respective Health facilities for cooperation and the permission of conducting the study was obtained from the head of each selected health facilities for the study.

\section{Informed consent}

Data was collected after assuring the confidentiality nature of responses and obtaining written consent from the study participant. All the study participants were encouraged to participate in the study and at 
the same time they were also told that they have the right not to participate.

\section{Dissemination of results}

The final finding of this study will be submitted and presented Bule Hora University. It will be also disseminated to West Guji Zone health office, and other concerned body and also will be tried to present at conferences and workshops. The finding of this study will be disseminated through different reputable journals and scientific publication

\section{Results}

\section{Socio-Demographic Characteristics of Respondents}

Out of a total 280 eligible Obstetrics care workers, 268 respondents were participated in this study with $95.7 \%$ response rate. About more than half $157(58.6 \%)$ of the respondents were in the age group of $20-$ 29 years; the mean age of respondents at which they attend Obstetric ward was 29.14 year with $\mathrm{SD} \pm 4.722$, ranging from 20 to 50 years. Nearly two third of the respondents, $172(64.2 \%)$ were married. One hundred sixty one(60.1\%) of the respondents were Nurses and 152(56.7\%) were degree holder; $199(74.3 \%)$ were male, and nearly half $124(46.3 \%)$ of the participants were protestants by religion. Two hundred fourteen $(79.9 \%)$ of them were Oromo in ethnicity (Table.3).

Table 2: Socio-demographic characteristics of Obstetric care workers, West Guji, June, 2021 


\begin{tabular}{|c|c|c|c|}
\hline Characteristics & Category & Number & Percentage \\
\hline \multirow{4}{*}{ Age in years } & $20-29$ & 157 & 58.6 \\
\hline & $30-39$ & 100 & 37.3 \\
\hline & $>=40$ & 11 & 4.1 \\
\hline & Total & 268 & 100 \\
\hline \multirow[t]{3}{*}{ Sex of respondents } & Male & 199 & 74.3 \\
\hline & Female & 69 & 25.7 \\
\hline & Total & 268 & 100 \\
\hline \multirow[t]{3}{*}{ Residence } & Urban & 189 & 70.5 \\
\hline & Rural & 79 & 29.5 \\
\hline & Total & 268 & 100 \\
\hline \multirow[t]{5}{*}{ Marital status } & Single & 82 & 30.6 \\
\hline & Married & 172 & 64.2 \\
\hline & Widowed & 10 & 3.7 \\
\hline & Divorced & 4 & 1.5 \\
\hline & Total & 268 & 100 \\
\hline \multirow{6}{*}{ Religion } & Muslim & 37 & 13.8 \\
\hline & Orthodox & 93 & 34.7 \\
\hline & Protestant & 124 & 46.3 \\
\hline & Catholic & 2 & .7 \\
\hline & Wakefata & 12 & 4.5 \\
\hline & Total & 268 & 100 \\
\hline \multirow[t]{6}{*}{ Ethnicity } & Oromo & 214 & 79.9 \\
\hline & Amhara & 40 & 14.9 \\
\hline & Tigre & 2 & .7 \\
\hline & Burji & 7 & 2.6 \\
\hline & Other & 5 & 1.9 \\
\hline & Total & 268 & 100 \\
\hline $\begin{array}{l}\text { Profession of the } \\
\text { respondents }\end{array}$ & Medical doctor & 12 & 4.5 \\
\hline
\end{tabular}




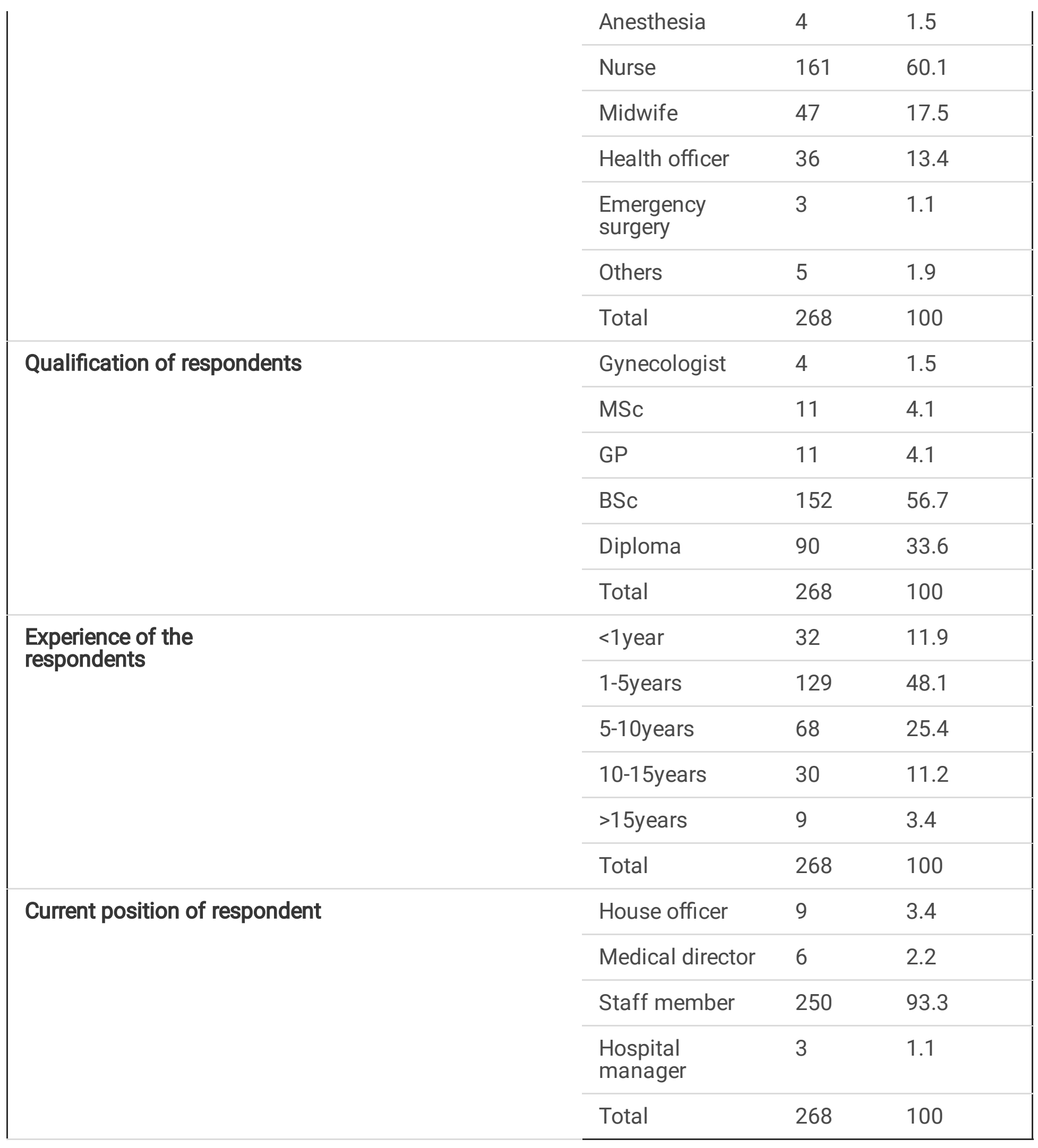

\section{Prevalence of Practice of labor pain management}

Information on labor pain management practice was collected from the obstetric care workers. Accordingly, from the total respondents, 116 (43.3\%) respondents used any form of labor pain management to clients for laboring mother, while those 152(56.7\%) were not (Fig2.) 


\section{Knowledge of Obstetric care workers related to labor pain management}

About 240 (89.6\%) participants had knowledge about labor pain management. Ninety eight( $40.8 \%)$ of them responded that they use non pharmacological method and $76(31.7 \%)$ of respondents were using both pharmacological and nonpharmacological methods and of nonpharmacological about $115(42.9 \%)$ respondents were used massage the back. About 66 (27.5\%) of study subjects are used pharmacological method. Of pharmacological method users 158(59\%) of them used intramuscular method.

\section{Attitude of Obstetric care workers related to Labor pain management}

Concerning the attitude of the respondents, more than half (53.7\%) of the study participants agreed that using labor pain influences the progress of labor and $145(54.1 \%)$ of respondents recommended that all mothers have to receive labor pain to relief the pain during labor, while $220(82.1 \%)$, of the respondents believed that labor pain is natural and mothers have to face it. About $209(78 \%)$ of respondents had thought of pharmacologic obstetric analgesia has effect on the labor and delivery outcomes, of these $116(55.5 \%)$ of study subjects think that it had the effect on labor outcome.About $118(43.3 \%)$ of respondents had the practice of labor pain management; of those of respondents who practiced labor pain management,nearly more than half $(52.6 \%)$ of them are used labor pain management for Episiotomy.And nearly $50 \%$ of these study participants were applying pharmacologic method.

\section{Institutional related factors of respondents}

The main institutional related reason that hindered from practicing labor pain management by respondents routinely were, $54(20.1 \%)$ of respondents had no reason, $145(54.1 \%)$ of study subjects complained that there is no drug, $12(4.5 \%)$ of them said that there is no equipment, $32(11.9 \%)$ of them inappropriate setting of the hospital, and $13(4.9 \%)$ of respondents had no knowledge/skill. Similarly, all of the participants responded that lack of trained health care providers, guidelines and protocols were the most common bottlenecks for utilizing the methods to relief labor pain.

\section{Factors associated with practice of labor pain management among respondent}

In bivariable analysis age of respondents, sex of respondents, current residence,experience of respondents,qualification of respondents,knowledge of obstetric analgesia,types of nonpharmacological method,thought of every mother should receive labor analgesia and thinking that pharmacologic obstetric has effect on the labor and delivery outcomes were showed significant association at $p<0.25$, but marital status of respondents did not show association. (Table 3)

Multivariable analysis revealed that: qualification of respondents, knowledge of obstetric analgesia and thought of pharmacologic obstetric analgesia has effect on the labor and delivery outcomeswere remained statistically significant of labor pain management practice at $p$-value $<0.05$. (Table 3 )

Being Diploma holder of obstetric care workers were 0.3 times less likely to practice labor pain management as compared to the counterparts (AOR, 0.3; $95 \% \mathrm{Cl}$ : 0.2,0.6). 
Respondents who had knowledge on obstetric analgesia were 9.4times more likely to practice labor pain management as compared to the referent (AOR, 9.4; 95\% Cl:1.8,9.9).

Obstetric care workers who had thought of pharmacologic obstetric analgesia has effect on the labor and delivery outcomeswere 2.9times more likely to practice labor pain management as compared to the referents (AOR, 2.9; 95\% Cl: 1.4,6.2).(Table 3).

Table 3:Bivariable and Multivariable logistic regression analysis of associated factors among Obstetric care workers, West Guji Zone, July 2021 


\begin{tabular}{|c|c|c|c|c|}
\hline \multirow[t]{3}{*}{ Characteristics } & \multicolumn{2}{|c|}{ Labor pain management practice } & \multirow[t]{3}{*}{$\operatorname{coR}(95 \% \mathrm{Cl})$} & \multirow[t]{3}{*}{$\mathrm{AOR}(95 \% \mathrm{Cl})$} \\
\hline & Yes & No & & \\
\hline & No.(\%) & No.(\%) & & \\
\hline \multicolumn{5}{|c|}{ Age of respondents } \\
\hline $20-29$ & $62(53.4 \%)$ & $95(62.5 \%)$ & $0.5(0.2,1.9)$ & $0.8(0.2,3.6)$ \\
\hline $30-39$ & $48(41.4 \%)$ & $52(34.2 \%)$ & $0.8(0.2,2.7)$ & $0.9(0.2,4.5)$ \\
\hline$=>40$ & $6(5.2 \%)$ & $5(3.3 \%)$ & 1 & \\
\hline \multicolumn{5}{|c|}{ Sex of respondents } \\
\hline Male & $93(80.2 \%)$ & 106(69.7\%) & $0.6(0.32,1.01)$ & $1.6(0.8,3.1)$ \\
\hline Female & 23(19.8\%) & $46(30.3 \%)$ & 1 & \\
\hline \multicolumn{5}{|l|}{ Residence } \\
\hline Urban & $91(78.4 \%)$ & $98(64.5 \%)$ & $2.0(1.2,3.5)$ & $1.7(0.9,3.2)$ \\
\hline Rural & $25(21.6 \%)$ & $54(35.5 \%)$ & 1 & \\
\hline \multicolumn{5}{|c|}{ Experience of respondents } \\
\hline$<1$ year & $10(8.6 \%)$ & $22(14.5 \%)$ & 1 & \\
\hline 1-5Years & $55(47.4 \%)$ & $74(48.7 \%)$ & $0.9(0.2,4.4)$ & $1.4(0.24,8.4)$ \\
\hline 5-10Years & $38(32.8 \%)$ & $30(19.7 \%)$ & $1.5(0.4,6.2)$ & $2.7(0.6,13.4)$ \\
\hline 10-15Years & $10(6.8 \%)$ & $20(13.2 \%)$ & $2.5(0.6,10.9)$ & $2.4(0.5,12.3)$ \\
\hline$>15$ years & $3(2.6 \%)$ & $6(3.9 \%)$ & $1.0(0.2,4.8)$ & $2.3(0.4,13.9)$ \\
\hline \multicolumn{5}{|c|}{ Qualification of respondents } \\
\hline Gynecologist & $1(0.9 \%)$ & $3(2 \%)$ & 1 & \\
\hline MSc & $3(2.6 \%)$ & $8(5.3 \%)$ & $0.2(0.02,2.12)$ & $0.9(0.3,2.7)$ \\
\hline GP & $7(6.0 \%)$ & $4(2.6 \%)$ & $0.24(0.6,0.96)$ & $1.8(0.9,3.5)$ \\
\hline BSc & $60(43.1 \%)$ & 102(67.1\%) & $1.1(0.3,4.1)$ & $1.6(0.7,3.6$ \\
\hline Diploma & $55(47.4 \%)$ & $35(23 \%)$ & $0.3(0.2,0.54)$ & $0.3(0.2,0.6) *$ \\
\hline \multicolumn{5}{|c|}{ Knowledge of obstetric analgesia } \\
\hline Yes & $113(97.4 \%)$ & $127(83.6 \%)$ & $7.4(2.2,25.2)$ & $9.4(1.8,9.9) *$ \\
\hline No & $3(2.6 \%)$ & $25(16.4 \%)$ & 1 & \\
\hline \multicolumn{5}{|c|}{ Types of nonpharmacological method } \\
\hline Acupuncture & $3(2.6 \%)$ & $\begin{array}{l}2(1.3 \%) \\
14 / 19\end{array}$ & 1 & \\
\hline
\end{tabular}




\begin{tabular}{|lcccc|} 
Diversional therapy & $5(4.3 \%)$ & $12(7.9 \%)$ & $4.8(0.64,15.5)$ & $7.9(0.8,8.9)$ \\
Psychotherapy & $51(41.4 \%)$ & $51(33.6 \%)$ & $1.3(0.3,5.3)$ & $1.7(0.4,7.8)$ \\
Massage the back & $49(42.2 \%)$ & $66(43.4 \%)$ & $2.9(1.1,8.1)$ & $3.5(1.2,10.1)$ \\
Hypnosis & $1(0.9 \%)$ & $1(0.7 \%)$ & $2.4(0.9,6.3)$ & $1.8(0.6,5.2)$ \\
Show the pt to bear down & $4(3.4 \%)$ & $1(0.7 \%)$ & $3.2(0.2,28.7)$ & $1.8(0.1,3.6)$ \\
Help to do labor exercises & $6(5.2 \%)$ & $19(12.5 \%)$ & $12.7(1.2,13.6)$ & \\
\hline Thought of every mother should receive labor analgesia & & $1.7(0.9,2.9)$ \\
\hline Yes & $74(63.8 \%)$ & $71(46.7 \%)$ & $2.0(1.2,3.3)$ & \\
No & $42(36.2 \%)$ & $81(53.3 \%)$ & 1 & $2.9(1.4,6.2) *$ \\
\hline Thought of pharmacologic obstetric analgesia has effect on the labor and delivery outcomes \\
\hline Yes \\
No & $101(87.1 \%)$ & $108(71.1 \%)$ & $2.7(1.4,5.2)$ & \\
\hline
\end{tabular}

\section{Note: * shows statistically significant at $P$ value $<0.05$}

\section{Discussion}

This study assessed the magnitude of Labor pain management practice and factors associated with labor pain management practice. Factors that associated with labor pain management practice were qualification of respondents, knowledge of obstetric analgesia and thought of pharmacologic obstetric analgesia has effect on the labor and delivery outcomes.

In this study, the magnitude of Labor pain management practice among Obstetric care workers was 43.3\%. This study finding was higher when compared to the study conducted KembataTembaro Zone, Southern and East Gojjam zone, Amhara region, Ethiopiawhich were $37.9 \%$ and $34.4 \%$ respectively $(27,36)$.The possible reason could be the effort of the health care workers at study areaand the time gap between the study conducted and the current study. This finding was higher as compared to the results of study conducted in Amhara regional state referral hospitals which was $40.1 \%$. The possible reason could be attributed to the scope of the study(35). This study finding was lower when compared to the study conducted in Zaria, Nigeria which was $48.4 \%(29)$. This discrepancy could be due to knowledge gap regarding labor pain management practice among health care workers between the regions and socioeconomic status may differs.

Having knowledge on obstetric analgesia were significantly associated to labor pain management practice in this study. In this study, respondents who had knowledge on obstetric analgesia were 9.4times more likely to practice labor pain management. This finding is consistent withstudy conducted in East Gojam zone, Amhara region, Ethiopia(19). 
Being Diploma holder of obstetric care workers were significantly associated to labor pain management practice in the current study. In this study, Obstetric are workers whose qualifications are at diploma level were $70 \%$ times more likely to practice labor pain management poorly. The finding of this is in line with the study conducted in Amhara regional state referral hospitals, northwest and General Hospitals in Tigray Region, North ,Ethiopia respectively $(35,37)$.

Having ideaof pharmacologic obstetric analgesia has effect on the labor and delivery outcomeswas significantly associated to labor pain management practice in the current study.

For this studyObstetric care workers who had thought of pharmacologic obstetric analgesia has effect on the labor and delivery outcomes were 2.9times more likely to practice labor pain management as compared to the referents. The finding of this study is comparable with the study conducted in University of Gondor, Amhara region, Ethiopia(34).

\section{Strength of the study}

As per the findings of this study, the authors recommend that labor pain management practice should be strengthened in all health facilities; providers should be given appropriate training and importance of labor pain management should be stressed during labor and delivery in order to improve the quality of deliveryprovision.

\section{Limitations of the study}

Since this study was a cross-sectional study design, the nature of the study may be difficult to ascertain the causal relationship between the study variables. As the study used was quantitative data collection method, it lacks the depth to identify the reasons behind of associated factors of early antenatal care booking.Since data collection was self-administered questionnaire, some incompleteness was faced.

\section{Conclusion}

The study showed that $43.3 \%$ of Obstetric care workers practice labor pain management. The factors that responsible for labor pain management practice were qualification of respondents, knowledge of obstetric analgesia and thought of pharmacologic obstetric analgesia has effect on the labor and delivery outcomes.

\section{Declarations}

\section{Acknowledgement}

We would like to forward our thanks to College of Health and Medical Sciences, Bule Hora University for giving us this chance to develop this research.Secondly, deepest gratitude goes to College of Health and Medical sciences research coordination office for the support to conduct the research. Our special thanks 
also forwarded to our instructors for their contribution of relevant information from inception of the title to the final work.

\section{Author Contributions}

Girma Tufa conceptualized the idea and wrote the original draft. ZelalemJabessaand Amana Jilosupervised the overall study and edited the whole document. Girma Tufa prepared the manuscript and all authors contributed to data analysis, drafting or revising the article.

\section{References}

1. FMOH. Labour and Delivery Care Blended learning module for health extension workers. 2015.

2. Jones L, Othman M, Dowswell T, Alfirevic Z, Gates S, Newburn M, et al. Pain management for women in labor: An overview of systematic reviews Pain management for women in labour. 2012;(March).

3. InternationalL A. Pain relief in Labor,forth edition of the Alarm internal program. 2011 p. 1-4.

4. Medicine C, Makvandi S, Mirzaiinajmabadi K, Tehranian N, Esmily H, Mirteimoori M, et al. The Effect of Normal Physiologic Childbirth on Labor Pain Relief: an Interventional Study in Mother-Friendly Hospitals. 2018;13(4):286-93.

5. WHO recommendation on epidural analgesia for pain relief during labour.

6. Global causes of maternal death: a WHO systematic analysis - The Lancet Global Health.

7. May $A E$, Elton $C D$. The effects of pain and its management on mother and fetus. Baillieres Clin Obstet Gynaecol. 1998 Sep;12(3):423-41.

8. Childbirth. Wikipedia. 2020.

9. Dobson M. Opinion: Labour Analgesia in the Developing World; Why Not. Rev Pain. 2011 Sep;5(3):23.

10. The Third Paradigm in Labour Pain Preparation and Management: The Childbearing Woman's Paradigm - PubMed.

11. Students' understanding of "Women-Centred Care Philosophy" in midwifery care through Continuity of Care (CoC) learning model: a quasi-experimental study | BMC Nursing | Full Text.

12. Med JB, Gagné G, Chaillet N, Rm RG, Rm EH, Buckley S, et al. No . 355-Physiologic Basis of Pain in Labour and Delivery: An Evidence-Based Approach to its Management. J Obstet Gynaecol Canada [Internet]. Elsevier Inc.; 2018;40(2):227-45. Available from:

https://doi.org/10.1016/j.jogc.2017.08.003

13. McCauley et al. - 2018 - "We know it's labour pain, so we don't do anything.pdf.

14. McCauley M, Actis Danna V, Mrema D, van den Broek N. "We know it's labour pain, so we don't do anything": healthcare provider's knowledge and attitudes regarding the provision of pain relief during labour and after childbirth. BMC Pregnancy Childbirth. 2018 Dec;18(1):444. 


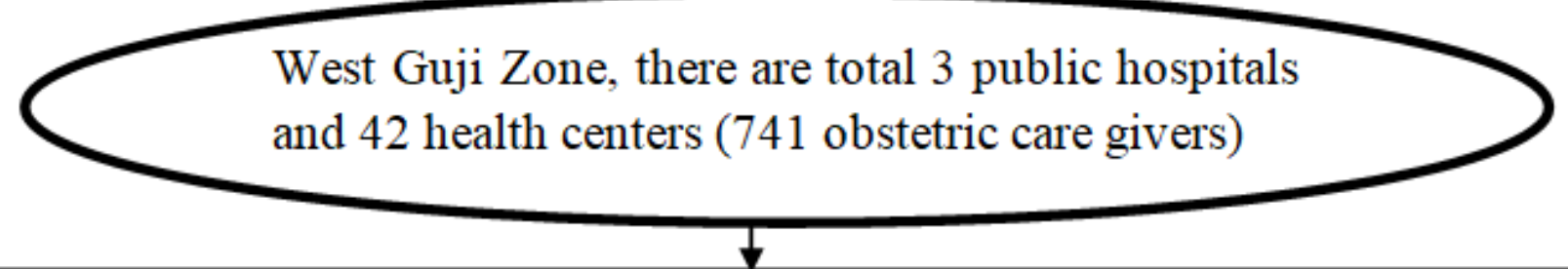

Among them all hospitals were included purposefully and $15 \mathrm{HC}$ will be randomly selected (491 obstetric care givers)

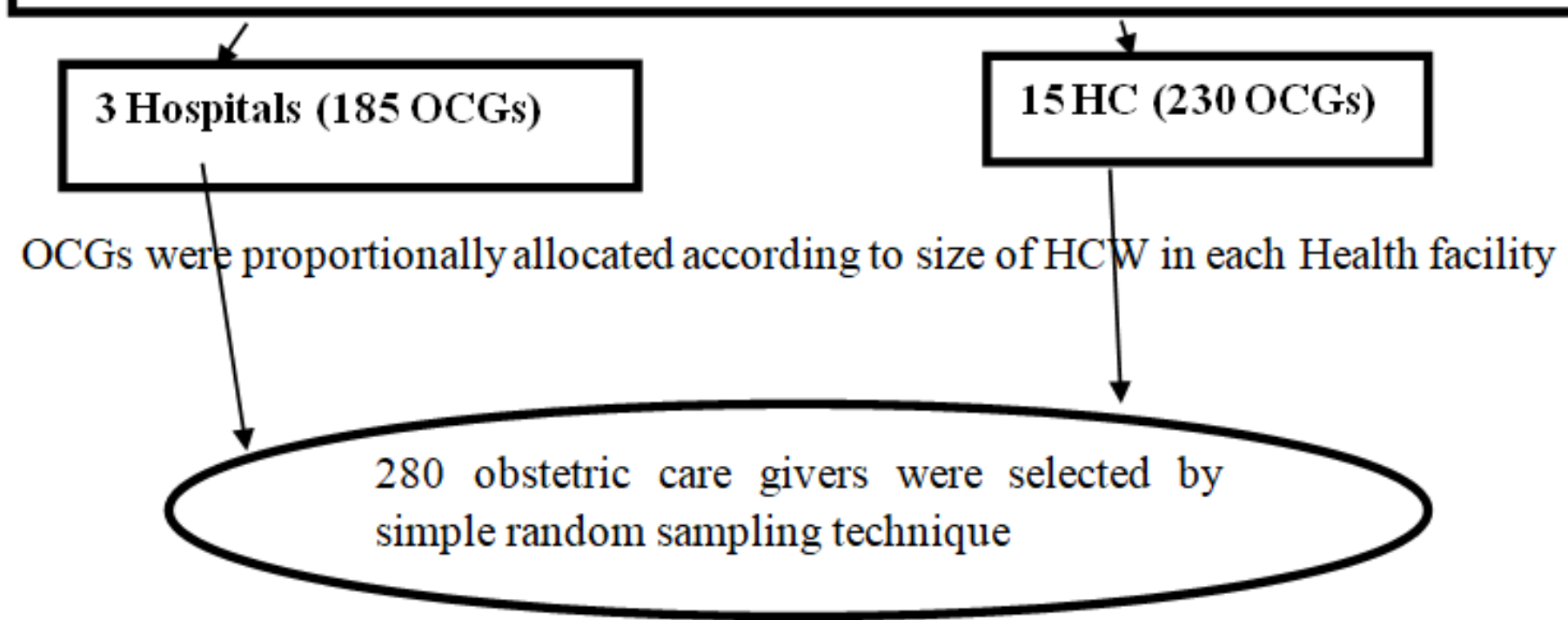

Figure 1

Shows proportional allocation of labor pain management practice among health care workers of west Guji Zone, 2020. 


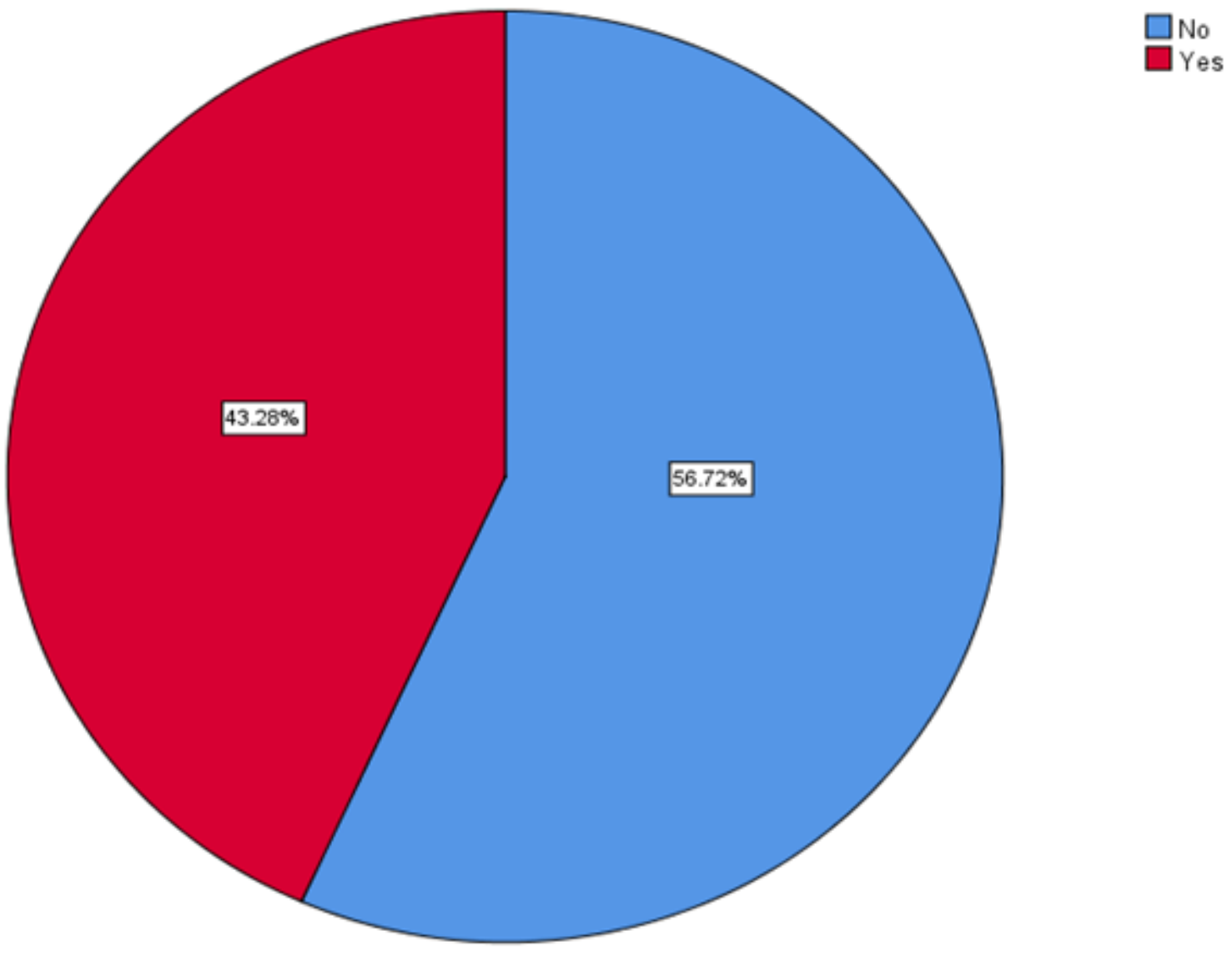

Figure 2

Shows the prevalence of labor pain management practice of the study area 